\title{
Prevalencia y factores de riesgo en Yucatán, México, para litiasis urinaria
}

\author{
Martha Medina-Escobedo, MC, ${ }^{(1)}$ Mussaret Zaidi, MC, ${ }^{(1)}$ Elizabeth Real-de León, Q, ${ }^{(2)}$
} Sergio 0 rozco-Rivadeneyra, MC. ${ }^{(3)}$

Medina-Escobedo M, Zaidi M, Real-de León E, Orozco-Rivadeneyra S. Prevalencia y factores de riesgo en Yucatán, México, para litiasis urinaria. Salud Publica Mex 2002;44:541-545.

El texto completo en inglés de este artículo está disponible en: http://www.insp.mx/salud/index.html

\section{Resumen}

Objetivo. D eterminar la prevalencia de urolitiasis en el estado deYucatán, México, y una posible asociación de ésta con antecedentes familiares de la enfermedad y la dureza del agua. Material y métodos D urante 1996 se efectuó una encuesta transversal en po blación abierta mayor de un año de edad, del estado de Yucatán. Los sujetos se clasificaron como litiasis definitiva (LD), litiasis probable (LP) y sin litiasis (SL). Se determinó dureza total, dureza de calcio y dureza de magnesio a las muestras de agua empleadas para el consumo humano. Se emplearon ji cuadrada y t de student para comparación de medias de muestras independientes en el análisis. Resultados. De un to tal de 5832 encuestas, 323 sujetos (5.5\%) se catalo garon como LD, y 282 (4.8\%) como LP. La prevalencia de litiasis se incremento con la edad, partiendo de $1 \%$ en población de 18 años o menor, hasta 11.3\% en los mayores de 50. El $44 \%$ del grupo LD y $34 \%$ del LP tuvieron antecedente familiar de urolitiasis, comparado con $28 \%$ del grupo SL $(p<0.0001$, RM 2.8, IC 95\% 2.2-3.4 y $p<0.0001$, RM 1.8, IC 95\%=1.4-2.4, respectivamente). Se observó que una dureza del agua mayor a 400 ppm se asocia con una mayor prevalencia de litiasis ( $p=0.003$, RM 1.9, IC 95\% 1.21-2.94). Conclusiones La prevalencia de litiasis urinaria en Yucatán es mayor a lo reportado en la literatura nacional e internacional. La dureza extrema del agua empleada para el consumo humano y el antecedente familiar de la enfermedad son factores de riesgo que requieren investigaciones futuras. El texto completo en inglés de este artículo está disponible en: http://www.insp.mx/salud/index.html

Palabras clave: cálculos urinarios; prevalencia; antecedente familiar; dureza del agua; México

\author{
Medina-Escobedo M, Zaidi M, Real-de León E, \\ Orozco-Rivadeneyra S. \\ Urolithiasis prevalence and \\ risk factors in Yucatan, Mexico. \\ Salud Publica Mex 2002;44:541-545. \\ The English version of this paper \\ is available at: http://www.insp.mx/salud/index.html
}

\begin{abstract}
A bstract
Objective To measure the prevalence of urolithiasis in Yucatan, and to establish a possible association of the disease with family history and water hardness. Material and Methods. During 1996, a survey was conducted on the population over one year of age in the State of Yucatan. Subjects were classified as having definite lithiasis (DL), probable lithiasis (PL), or no lithiasis (NL). Total hardness, calcium hardness, and magnesium hardness, were determined in drinking water samples. Results From a total of 5832 questionnaires, 323 subjects (5.5\%) were fo und to have DL, and $282(4.8 \%)$ PL. The prevalence of lithiasis increased sharply with age, ranging from $1 \%$ in the po pulation 18 years or yo unger, to $11.3 \%$ in those over 50 years.A family history of urolithiasis was positive in $44 \%$ of subjects with $D L$ and in $34 \%$ of those with PL, compared to $28 \%$ of those with $\mathrm{NL}(\mathrm{OR}=2.8,95 \% \mathrm{Cl}=2.2-3.4, \mathrm{p}<0.0001$, and $\mathrm{OR}=1.8,95 \%$ $C I=1.4-2.4, p<0.0001$, respectively). W ater hardness above $400 \mathrm{ppm}$ was associated with a higher prevalence of urolithiasis $(O R=1.9,95 \% C l=1.21-2.94, p=0.003)$. Conclusions The prevalence of urolithiasis in Yucatan is higher than that reported elsewhere in the scientific literature. A family history of the disease and high water hardness are risk factors that need to be investigated in future studies. The English version of this paper is available at: http://www.insp.mx/salud/index.html
\end{abstract}

Key words: urinary calcule; prevalence; family history; water hardness; Mexico

Este estudio fue financiado por la Fundación Mexicana para la Salud Capítulo Peninsular, y presentado en forma de resumen en el "Segundo Encuentro N acional de Investigadores de la Secretaría de Salud", en Huatulco, 0 axaca, México, el 18 de noviembre de 1997.

(1) D epartamento de Investigación. Hospital G eneral 0 'Horán. Mérida,Yucatán, México.

(2) Departamento de Recursos del Mar. Centro de Investigaciones y Estudios Avanzados, Unidad Mérida. Mérida, Yucatán, México.

(3) Secretaría A cadémica, Instituto N acional de Salud Pública, Cuernavaca, Morelos, México.

Fecha de recibido: 30 de enero de 2001 - Fecha de aprobado: 7 de junio de 2002

Solicitud de sobretiros: MC. Martha Medina-Escobedo. Departamento de Investigación, Hospital General 0 'Horán. Avenida Itzaes por Jacinto Canek. 97000. Mérida,Yucatán, México.

Correo electrónico: marthamedes@ hotmail.com 
a urolitiasis es una enfermedad multifactorial en la que se han implicado aspectos epidemiológicos, raciales, geográficos y hereditarios de las poblaciones estudiadas. ${ }^{1}$ La prevalencia mundial varía de cuatro a 17 casos $/ 1000$ habitantes. ${ }^{2}$ Se describen como factores de riesgo para urolitiasis hipercalciuria, hiperuricosuria, el volumen urinario, la dieta y los factores genéticos, entre otros., ${ }^{3,4}$

El impacto económico de la enfermedad es considerable debido a la recurrencia de infecciones urinarias, a la necesidad de extracción quirúrgica o litrotipsia y, en el peor de los casos, a la progresión hacia la insuficiencia renal crónica. ${ }^{5-7}$

En México se han efectuado pocos estudios epidemiológicos de la urolitiasis. Otero y colaboradores ${ }^{8}$ reportaron que este padecimiento comprende $13 \%$ de todas las hospitalizaciones por enfermedad renal en el ámbito nacional en el Instituto Mexicano del Seguro Social (IMSS). Otra encuesta nacional efectuada en este mismo Instituto reportó una prevalencia de 2.4 casos de urolitiasis/10 000 habitantes derechohabientes, y menciona a los estados de Yucatán, Puebla y Quintana Roo como áreas endémicas, de las cuales Yucatán tuvo la prevalencia más elevada con 5.8 casos/ 10000 derechohabientes. ${ }^{9}$

La experiencia en el Hospital General O'Horán, hospital de referencia de tercer nivel de atención en Yucatán, sugiere que la prevalencia de urolitiasis es más alta que la referida en otras regiones. En este nosocomio se observan 46 casos nuevos de urolitiasis por año; asimismo, $77.6 \%$ de los pacientes con diagnóstico de urolitiasis requieren de tratamiento quirúrgico para la solución parcial de su problema y $87 \%$ tiene alguna complicación relacionada con urolitiasis, ${ }^{5}$ de manera que esta enfermedad y sus complicaciones son más comunes que las glomerulopatías, lo que contrasta claramente con la experiencia de otros hospitales en México, en donde son más frecuentes estas últimas..$^{6,8}$

El elevado porcentaje de admisiones hospitalarias, procedimientos quirúrgicos y serias complicaciones relacionadas con la urolitiasis subraya la necesidad de investigar este problema de salud en Yucatán.

El papel de la dureza del agua en el desarrollo de litiasis urinaria es aún motivo de controversia. Conviene señalar que aquellos estudios que reportan que la dureza del agua no contribuye al desarrollo de la litiasis urinaria se hicieron en comunidades en las que la dureza total no superaba las $400 \mathrm{ppm} .{ }^{10,11}$ Por el contrario, la Comisión Nacional del Agua (México) reconoce determinaciones de durezas totales por arriba de 800 ppm en el agua empleada para consumo humano, en algunas comunidades del estado de Yucatán. ${ }^{12}$
Por lo anterior, se decidió aplicar una encuesta para determinar la prevalencia de urolitiasis en la población del estado, y evaluar los antecedentes familiares y la dureza elevada del agua como posibles factores de riesgo para el desarrollo de la enfermedad.

\section{Material y métodos}

Se empleó un diseño de encuesta transversal; basándose en los resultados de una prueba piloto, se calculó un tamaño de muestra de 5703 individuos correspondientes a una prevalencia de $3 \%$, una taza de respuesta de $90 \%$ y un límite de confianza de $95 \%$. Se eligieron al azar 90 localidades (lo que corresponde a 18\% de todas las localidades con más de 100 habitantes en el estado) y se determinó el municipio al que correspondía cada una de ellas. Las 5703 encuestas fueron asignadas a cada una de las comunidades de acuerdo con la proporción de habitantes registrada en ellas, según datos obtenidos del Censo General de Población de 1990. ${ }^{13}$ Para el análisis de la dureza del agua se clasificaron los municipios del estado de Yucatán en tres estratos: estrato 1, "dureza desconocida"; estrato 2, "dureza intermedia" (300-400 ppm), y estrato 3, "dureza elevada" ( $\geq 401 \mathrm{ppm})$. Cada poblado se dividió en cuatro sectores iguales y las casas elegidas para las entrevistas fueron seleccionadas en cada sector en forma aleatoria. De enero a septiembre de 1996, previo entrenamiento, cinco trabajadoras sociales visitaron cada casa seleccionada. En cada una se incluyeron todos los sujetos mayores de un año de edad, se aplicó un cuestionario para consignar datos sobre sexo, edad, antecedentes personales y familiares de urolitiasis, y tipo de agua consumida.

Definiciones. A los sujetos con antecedente de haber expulsado un cálculo, o haber sido intervenido para la extracción de un cálculo urinario se les clasificó como litiasis definitiva (LD). En la categoría de litiasis probable (LP), considerada una forma subclínica de la enfermedad, ${ }^{7,14}$ se incluyeron aquellos sujetos con abundante sedimento urinario acompañado por disuria o hematuria. Por último, en el grupo sin litiasis (SL) se incluyeron aquellos sujetos que no tuvieron ningún criterio de las otras categorías.

En el interior del estado se tomaron por duplicado de cuatro a cinco muestras de la tubería que provenía de la fuente de abastecimiento de agua potable en cada comunidad encuestada. En la ciudad de Mérida se consideró la localización de los pozos de abastecimiento de agua potable para la población, de donde se tomaron 24 muestras para análisis. Las muestras de agua de pozo se tomaron de los domicilios en los cuales 
se refirió consumo de este tipo de agua. Las muestras se tomaron en frascos de polivinilo, previamente lavados con el agua de la llave de donde se iba a tomar la muestra, y se mantuvieron a $4{ }^{\circ} \mathrm{C}$ hasta su análisis; luego de su colección fueron transportadas y analizadas dentro de un periodo no mayor a 24 horas. Todas las muestras de agua se enviaron al Laboratorio Estatal de la Comisión Nacional del Agua y al Departamento de Recursos del Mar del Centro de Investigaciones y Estudios Avanzados (Cinvestav)/Unidad Mérida. Los métodos empleados para la determinación de la dureza total (DT), la dureza de calcio (DCa) y la dureza de magnesio (DMg), se han descrito previamente..$^{15}$

El análisis estadístico empleado para evaluar el antecedente familiar de litiasis fue la ji cuadrada; la prueba de $t$ de Student se utilizó para la comparación de medias de muestras independientes de agua de pozo y agua potable. La asociación de LD con DT, DCa y DMg de cada una de las poblaciones encuestadas, se hizo de manera inicial de acuerdo con la dureza determinada por estratos y, posteriormente, considerando dos niveles de dureza del agua: poblaciones con dureza $\leq$ a $400 \mathrm{ppm}$ y poblaciones con dureza $\geq$ 401 ppm. Se estableció el número de casos con LD y se efectuó el análisis comparativo entre los grupos mediante ji cuadrada. Se determinó la razón de momios con un intervalo de confianza de $95 \%$ para establecer las inferencias. El nivel de significancia para la $p$ se consideró en 0.05 .

\section{Resultados}

Se aplicaron 5832 encuestas, de las cuales 3656 fueron a mujeres, y 2176 a hombres. Se detectaron 323 casos de LD (5.5\%) y 282 de LP (4.8\%); no hubo predominio geográfico. El $5.2 \%$, 191 mujeres y 132 hombres (6\%) tuvieron LD, y 216 mujeres (5.9\%) y 66 hombres (3\%) tuvieron LP. Estas diferencias no fueron estadísticamente significativas $(p>0.05)$. La prevalencia se incrementó en forma significativa con la edad, partiendo de $1 \%$ en sujetos menores de 18 años hasta $11.3 \%$ en aquellos mayores de 50 años $(p<0.0001){ }^{16}$ (cuadro I).

El $44 \%$ de los sujetos con LD y $34 \%$ de los sujetos con LP tuvieron historia familiar de litiasis, comparado con $28.1 \%$ de los sujetos SL $(p<0.0001, R M=2.8$, IC $95 \%=2.2-3.4$ y $p<0.0001, R M=1.8$, IC $95 \%=1.4-2.4$, respectivamente).

En el cuadro II se muestra la prevalencia del antecedente familiar único en el padre, la madre o hermanos de los sujetos con LD, comparado con los sujetos SL; la prevalencia fue mayor en los hermanos $(p<0.0001$, RM 2.6, IC 95\%=1.83-3.80). Se observó un patrón similar en el grupo LP (cuadro III).

Se analizaron 374 muestras de agua, 29 de ellas correspondieron a agua de pozo y las restantes a muestras de agua potable. Los resultados de DT, DCa, y $\mathrm{DMg}$, de ambos laboratorios participantes, no difirieron en más de $10 \%$, por lo que se empleó la media de estos valores para el análisis estadístico. No se observó diferencia entre las medias de la DT, DCa y DMg al comparar el agua de pozo con el agua potable $(p>0.05)$. Los rangos de dureza observados considerando todas las muestras de agua, fueron DT 336-1321 ppm, DCa 216-885 ppm y DMg 44-436 ppm. El análisis no mostró diferencia significativa en DT, DCa y DMg al comparar las medias de dureza entre los estratos I, III y el estrato II, por lo que se decidió tomar a los poblados con DT $£$ a 400 ppm como grupo I, y a los que tuvieron DT $\geq 401$ ppm como grupo II, para efectuar el análisis comparativo tomando en cuenta la prevalencia de LD (cuadro IV).

La prevalencia de LD en el grupo I (DT $£$ a 400 ppm) fue de $3.3 \%$, comparada con $6.21 \%$ en el grupo II (DT $\geq 401 \mathrm{ppm}), p=0.003, R M=1.88$, IC $95 \%$ 1.21-2.94.

\section{Cuadro I \\ Prevalencia Y RIEsgo de litiasis definitiva Y litiasis Probable de acuerdo Con los Grupos de edad en YuCatán, MéXICo,* 1996}

\begin{tabular}{|c|c|c|c|c|c|c|c|c|c|c|}
\hline \multirow[t]{2}{*}{ Resultados } & \multicolumn{2}{|c|}{$<18$ años ( $n=2062)$} & \multicolumn{2}{|c|}{19 a 30 años ( $n=1228)$} & \multicolumn{2}{|c|}{31 a 40 años $(n=890)$} & \multicolumn{2}{|c|}{41 a 50 años $(n=682)$} & \multicolumn{2}{|c|}{$>50$ años $(n=970)$} \\
\hline & $\%$ & RM & $\%$ & RM & $\%$ & RM & $\%$ & & $\%$ & \\
\hline Litiasis definitiva (LD) $)^{\ddagger}$ & 1.0 & 1 & 4.7 & 4.1 & 8.3 & 7.4 & 8.9 & 8.1 & 11.3 & 11.1 \\
\hline Litiasis probable (LP) ${ }^{\ddagger}$ & 1.2 & 1 & 6.7 & 6.3 & 6.8 & 5.8 & 9.9 & 10.2 & 5.3 & 5.3 \\
\hline
\end{tabular}




\section{Cuadro II \\ Antecedente familiar positivo en pacientes Con litiasis definitiva en Yucatán, México,* 1996}

Familiar afectado Litiasis definitiva $\underset{(n=323)}{\operatorname{Sin} \text { litiasis }} \underset{(\mathrm{n}=5227)}{\mathrm{Ji}^{2}} \quad \mathrm{RM}^{\ddagger} \neq \quad$ IC $95 \%$ $(\%) \quad(\%)$

\begin{tabular}{llllll} 
Padre & 10.8 & 4.7 & $<0.0001$ & 2.4 & $1.63-3.56$ \\
\hline Madre & 13.0 & 5.1 & $<0.0001$ & 2.7 & $1.91-3.93$ \\
\hline Hermanos & 18.2 & 6.0 & $<0.0001$ & 3.4 & $2.53-4.75$
\end{tabular}

* Encuestas efectuadas de enero a septiembre de 1996

‡ Razón de momios cruda

Nota: algunos sujetos tuvieron el antecedente de litiasis en más de un familiar, por lo que el total de sujetos con algún antecedente es mayor

\section{Cuadro III \\ Antecedente familiar POSITIVo EN PACIENTES CON Litiasis PRobable en YuCatán, MéXICO,* 1996}

$\begin{array}{ccccc}\text { Familiar afectado } & \begin{array}{ccc}\text { Litiasis probable } \\ (\mathrm{n}=282)\end{array} & \begin{array}{c}\text { Sin litiasis } \\ (\mathrm{n}=5227)\end{array} & \mathrm{Ji}^{2} & (\mathrm{p})\end{array} \quad \mathrm{RM}^{\ddagger} \quad$ IC $95 \%$

\begin{tabular}{lrrrrr} 
Padre & 9.9 & 4.7 & $<0.0001$ & 2.2 & $1.42-3.36$ \\
\hline Madre & 10.2 & 5.1 & 0.0003 & 2.1 & $1.37-3.20$ \\
\hline Hermanos & 14.5 & 6.0 & $<0.0001$ & 2.6 & $1.83-3.80$
\end{tabular}

* Encuestas efectuadas de enero a septiembre de 1996

₹ Razón de momios cruda

Nota: algunos sujetos tuvieron el antecedente de litiasis en más de un familiar, por lo que el total de sujetos con algún antecedente es mayor

\section{Discusión}

La prevalencia de LD detectada en este estudio (5.5\%) es de 10 a 100 veces más alta que la reportada en la literatura médica. ${ }^{1,9,17}$ Si se incluyen los casos de LP, (considerados como una forma subclínica de la enfermedad) ${ }^{7,14}$ la prevalencia real de urolitiasis en el estado de Yucatán sería de 10.3\%. No se observó en el estado predominio geográfico para litiasis, sin embargo esto deberá ser confirmado en estudios futuros.

La prevalencia de LD en sujetos mayores de 50 años fue 11 veces más elevada que en aquellos menores de 18 años $(p<0.0001, R M=11.17)$. El hecho de que la prevalencia se incremente en forma notable con la edad sugiere que la urolitiasis puede desarrollarse posterior a la exposición repetida a un factor externo. Uno de los pocos estudios que muestra una prevalencia elevada comparable se efectuó en Kaizuka, Japón;

\section{Cuadro IV \\ Comparación entre LAS MEdias de duREza total, DUREZA DE CALCIO, DUREZA DE MAGNESIO Y RELACIÓN MAGNESIO:CALCIO DE LOS GRUPOS ESTUDIADOS. Yucatán, México, 1996}

$\begin{array}{lccc}\text { Variable } & \text { Grupo I } & \text { Grupo II } & p \\ & \text { (DT } \leq 400 \mathrm{ppm}) & (\mathrm{DT} \geq 401 \mathrm{ppm}) & \text { (p) }\end{array}$

\begin{tabular}{lccc} 
Dureza de calcio & $279 \pm 31$ & $337 \pm 91$ & $<0.0001^{*}$ \\
\hline Dureza de magnesio & $99 \pm 25$ & $172 \pm 77$ & $<0.0001^{*}$ \\
\hline Relación Mg:Ca & $0.36 \pm 0.13$ & $0.50 \pm 0.17$ & $<0.0001^{*}$ \\
\hline Prevalencia de litiasis definitiva & $3.3 \%$ & $6.2 \%$ & $<0.003^{\ddagger}$
\end{tabular}

* t de student a dos colas

₹ $p$ de ji2 con corrección de Yates

los autores detectaron una prevalencia de $4.5 \%$ en población general, la cual también se incrementaba con la edad, de modo que $10.3 \%$ de los sujetos mayores de 50 años padecen la enfermedad, lo cual es muy similar a nuestros hallazgos. ${ }^{18}$

En este estudio un factor de riesgo importante para LD y LP fue el antecedente de urolitiasis en padres o hermanos de los sujetos afectados. Estudios previos han hallado una forma de transmisión autosómica dominante en pacientes con hipercalciuria idiopática ${ }^{19,20}$ en quienes la urolitiasis es relativamente común. Asimismo, se sabe de la gran influencia que tienen los antecedentes familiares de padres y hermanos, en los pacientes con cálculos urinarios en las vías urinarias superiores. ${ }^{21}$

Las diferencias observadas entre la prevalencia de litiasis en las poblaciones, utilizando como punto de corte una dureza del agua de 400 ppm, apoyan la hipótesis de que esta dureza incrementa la probabilidad de padecer litiasis urinaria cuando rebasa este límite. Numerosas investigaciones han tratado de establecer una relación entre las características físico-químicas del agua y la urolitiasis, sin encontrar asociación. Un motivo aparente para la falta de asociación es que estos estudios se efectuaron en comunidades donde la dureza del agua empleada para consumo humano no alcanzaba el límite establecido en este estudio (400 ppm). En la región de Rockie la media de la DT fue 58 ppm, en la región de Carolina la DT fue de $13.3 \mathrm{ppm}$, comparada con nuestra media de DT de 485 ppm. ${ }^{11,22}$

Aunque la Organización Mundial para la Salud no tiene valores de referencia recomienda 200 ppm como límite aceptable en la dureza total del agua empleada para consumo humano y 500 ppm como límite 
permisible. ${ }^{23}$ La Norma Oficial Mexicana refiere como límite máximo $200 \mathrm{ppm} \cdot{ }^{24} \mathrm{La}$ dureza total de todas las muestras de agua obtenidas en nuestro estudio excedió el límite aceptable establecido nacional e internacionalmente $(200 \mathrm{ppm})$ y $20 \%$ de las muestras rebasaron el límite de tolerancia (500 ppm).

Por otra parte, Kohri encontró una asociación positiva entre la relación magnesio/calcio del agua empleada para consumo humano y la prevalencia de los cálculos renales de estruvita en regiones de basalto y roca sedimentaria en Japón. ${ }^{25}$ Asimismo, Bellizzi y colaboradores observaron un incremento significativo, hasta de $50 \%$, en la concentración de calcio urinario en ausencia de cambios en la excreción de oxalato, y un aumento hasta tres veces mayor en el índice calcio/citrato durante la ingestión de agua con dureza elevada (255ppm) cuando se comparó con la ingesta de agua blanda (22ppm) ${ }^{26}$

Por lo anterior, pese a la controversia sobre la influencia del agua en la formación de la litiasis urinaria, nuestros datos apoyan el hecho de que la dureza extrema del agua es un factor predisponente para el desarrollo de la enfermedad.

\section{Conclusiones}

La prevalencia de urolitiasis en el estado de Yucatán es elevada y se incrementa en forma notable con la edad. El antecedente familiar y la dureza extrema ( $\leq 400$ ppm) del agua empleada para consumo humano son factores predisponentes para el desarrollo de la enfermedad.

Se requieren investigaciones futuras para definir la magnitud del impacto de estos factores y el papel de otros solutos en el desarrollo de urolitiasis.

\section{A gradecimientos}

Los autores agradecen al ingeniero Fernando Ponce García por el apoyo brindado para que este estudio fuese llevado a cabo.

\section{Referencias}

1. Scott R. Epidemiology of stone disease. Br J U rol 1985;57:491-497. 2. Curhan C, Rimm E,W illet W, Stampfer M. Regional variation in nephrolithiasis incidence and prevalence among United States men. J Urol 1994;151:838-841.
3. Robertson W, Peacok M, Heyburn P, Hanes F. Epidemiological risk factors in calcium stone disease. En: Danielson BG, ed. Proceedings of the Symposium in urolithiasis. U ppsala:W ellcome Foundation, 1980: 15-28.

4. Danpure Ch. Genetic disorders and urolithiasis. Ur Clin North Am 2000;27(2):287-299.

5. C anul G. Evaluación clínica de la litiasis urinaria observada en el Hospital G eneral 0 'H orán, S.S.A. Yucatán, Universidad Autónoma de Yucatán: 1997.

6. Gordillo G. Insuficiencia renal crónica. En: Gordillo G, ed. N efrología pediátrica. México, D.F.: Mosby-D oyma, 1996:374-400.

7. Maffly R. VIII N efrolitiasis. En: Rubenstein E, Federman D, ed. Scientific A merican Medicine. México, D.F. Scientific American, 1988;vol.2: 1-9.

8. 0 tero $F$, Lugo A, Durán A. Las enfermedades renales en el Instituto Mexicano del Seguro Social (1982-1989). Rev Asoc Med Int Mex 11;1995: 21-29.

9. Gómez F, Reyes G, Espinosa L,Arellano H, Morales M, Gómez R. Algunos aspectos epidemiológicos de la litiasis renal en México. C irugia y C irujanos 1984;52:365-372.

10.Assimos D, Holmes R. Role of diet in the therapy of urolithiasis. U rol Clin N orth Am 2000;27(2):255-268.

11. Shuster J, Finlayson B, Scheaffer R, Sierakowski R, Zoltek J,D zegedes. W ater hardness and urinary stone disease. J U rol 1982;126:422-425.

12.Vidal J. Boletín de calidad del agua. Mérida,Yucatán:C omisión N acional del Agua, 1994.

13. Instituto N acional de Estadística, Geografía e Informática. Resultados definitivos. Datos por localidad (integración ter ritorial).Yucatán.XI Curso General de Población y Vivienda, 1990. México, D.F.: IN EGI, 1993. 14. Alon $U, W$ arady BA, Hellerstein $S$. Hypercalciuria in the frecuencydysuria syndrome of chilhood. J Pediatr 1990;116: 103-105.

15. A merican Public Health Association. Standard methods for the examination of water and wastewater. 19th Edition. W ashington, D.C :. APHA, 1995.

16. Schlesseman J. C ase-control studies. N uevaYork (N Y): 0 xford University Press 1982:203-206.

17. Sriboonlue P, Prasongwatana V, Chata K, Tungzanga K. Prevalence of upper urinary tract stone disease in a rural community of $\mathrm{N}$ orth-Eastern Thailand. Br J U rol 1992;69:240-244.

18. Iguchi M, U mekawa T, Katoh Y, Kohri K, Kurita T. Prevalence of urolithiasis in Kaizuka C ity, Japan:An epidemiologic study of urinary stones. Int J Urol 1996;3:175-179.

19.W einberger A, Schecheter J, Pinkhas J, Sperling 0. Hereditary hypercalciuric urolithiasis. Br J U rol 1981;53:285-286.

20. Dumas R. Hypercalciuria: Etiologies and treatment. Arch Pediatr 1997:44:351-358.

21. Ishikawa Y, Konya E, Yamate T. Influence of genetic factors on family history of upper urinary stones. Hinyokika Kiyo 1995;41(5):349-353.

22. Singh P, Kiran R. Are we overstressing water quality in urinary stone disease? Int Urol N ephrol 1993;25(1):29-36.

23. W orld Health $O$ rganization. Guidelines for D rinking-W ater Q uality. Vol. 1: Recommendations. Ginebra:W HO, 1993.

24. N orma 0 ficial Mexicana N O M-041-SSA 1-1993, Bienes y Servicios.A gua purificada envasada. Especificaciones sanitarias. Diario 0 ficial de la Federación CDXCVIII 18, 24 de marzo, México, 1995;14-15.

25. Kohri K, Ishinkawa Y, Iguchi M. Relationship between the incidence infection stones and the magnesium-calcium ratio of tap water. U rol Res 1993;21(4):269-272.

26. Bellizi V, De Incola L, Minutolo R, Russo D, C ianciaruso B,Andreucci M et al. Effects of water hardness on urinary risk factors for kidney stones in patients with idiopathic nephrolithiasis. N ephron 1999;81(suppl 1):66-70. 